\title{
The edge wave on an elastically supported Kirchhoff plate (L)
}

\author{
Julius Kaplunov, Danila A. Prikazchikov, ${ }^{\text {a) }}$ and Graham A. Rogerson \\ School of Computing and Mathematics, Keele University, Keele, Staffordshire, ST5 5BG, United Kingdom \\ Mohamed I. Lashab \\ Faculty of Science, University of Almergeb, Zliten, Libya
}

(Received 7 January 2014; revised 18 July 2014; accepted 16 August 2014)

\begin{abstract}
This Letter deals with an analysis of bending edge waves propagating along the free edge of a Kirchhoff plate supported by a Winkler foundation. The presence of a foundation leads to a nonzero cut-off frequency for this wave, along with a local minimum of the associated phase velocity. This minimum phase velocity corresponds to a critical speed of an edge moving load and is analogous to that in the classical 1D moving load problem for an elastically supported beam. (C) 2014 Acoustical Society of America. [http://dx.doi.org/10.1121/1.4894795]
\end{abstract}

PACS number(s): 43.40.Dx, 43.20.Jr [MDV]

Pages: $1487-1490$

\section{INTRODUCTION}

Edge bending waves in respect of a free elastic plate are well-studied within the framework of low frequency classical theories of 2D thin plates, often referred to as Kirchhoff plates, see Refs. $1-3$, and references therein.

In this Letter we extend previous considerations to investigate a Kirchhoff plate resting on a Winkler foundation, i.e., when the reaction of the foundation is directly proportional to the deflection of the plate, e.g., see Ref. 4. The model of a plate supported by a Winkler foundation has a number of important applications, in particular, in structural mechanics. ${ }^{5}$

In spite of having a dispersion relation similar to that of a free Kirchhoff plate, the presence of a foundation brings a number of novel features, including a local minimum of the edge wave phase velocity which in fact coincides with the related group velocity. This minimum also coincides with the critical wave speed of an edge moving load, as in the classical steady-state problem for a beam on an elastic foundation. ${ }^{6}$

\section{GOVERNING RELATIONS}

Consider a Kirchhoff elastic plate of thickness $2 h$ resting on a foundation of Winkler type ${ }^{4}$ (Fig. 1). The deflection of the plate $W(x, y, t)$ is then governed by

$$
D \Delta^{2} W+2 \rho h \frac{\partial^{2} W}{\partial t^{2}}+\beta W=0,
$$

where $\Delta=\partial^{2} / \partial x^{2}+\partial^{2} / \partial y^{2}$ is the $2 \mathrm{D}$ Laplace operator, $\rho$ is the volume density of mass, $\beta$ is the Winkler constant, and $D$ is bending stiffness, given by

$$
D=\frac{2 E h^{3}}{3\left(1-\nu^{2}\right)},
$$

with $E$ and $\nu$ denoting the Young modulus and the Poisson ratio, respectively. We remark that the final term of Eq. (1) is present because of the existence of the Winkler

\footnotetext{
a) Author to whom correspondence should be addressed. Electronic mail: d.prikazchikov@keele.ac.uk
}

foundation, $\beta=0$ corresponding to the classical Kirchhoff plate problem, see, e.g., Ref. 7.

The boundary conditions at the free edge $y=0$ are taken in the form ${ }^{1}$

$$
\begin{aligned}
& \frac{\partial^{2} W}{\partial y^{2}}+\nu \frac{\partial^{2} W}{\partial x^{2}}=0, \\
& \frac{\partial^{3} W}{\partial y^{3}}+(2-\nu) \frac{\partial^{3} W}{\partial x^{2} \partial y}=0 .
\end{aligned}
$$

\section{DISPERSION ANALYSIS}

Following a now standard procedure for derivation of the dispersion relation, the deflection of the plate is sought for in the form of a traveling harmonic wave, propagating in the positive direction of $x$,

$$
W(x, y, t)=A e^{i(k x-\omega t)-k \lambda y},
$$

where $k \geq 0$ and $\omega \geq 0$ are the wave number and frequency, respectively. Here $\lambda$ is the attenuation coefficient, with the condition $\operatorname{Re}(\lambda)>0$ ensuring that the wave decays away from the edge $y=0$ of the plate $(y \geq 0)$. Substitution of Eq. (5) into the plate equation (1) leads to a bi-quadratic equation in $\lambda$, taking the form

$$
\lambda^{4}-2 \lambda^{2}+1-\frac{2 \rho h \omega^{2}-\beta}{k^{4} D}=0 .
$$

It may be shown that two of the roots of Eq. (6) satisfy the decay conditions $\operatorname{Re}(\lambda)>0$, hence, the solution of Eq. (1) may be expressed as

$$
W(x, y, t)=\sum_{j=1}^{2} C_{j} e^{i(k x-\omega t)-k \lambda_{j} y},
$$

with the associated attenuation orders $\lambda_{1}$ and $\lambda_{2}$ given by

$$
\lambda_{1}=\sqrt{1-\frac{k_{0}^{2}}{k^{2}}},
$$




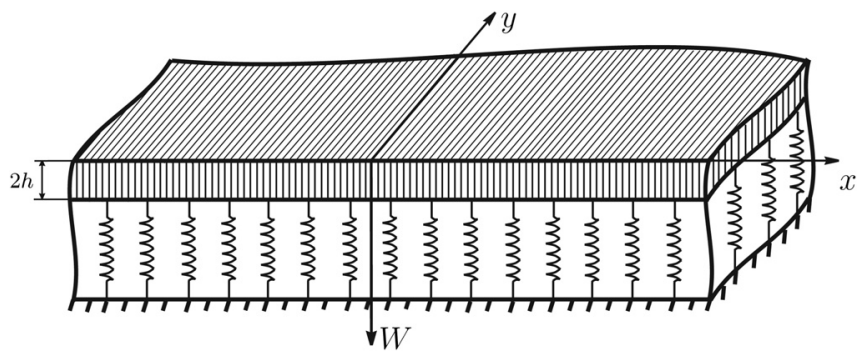

FIG. 1. Elastically supported plate.

$$
\lambda_{2}=\sqrt{1+\frac{k_{0}^{2}}{k^{2}}}
$$

where

$$
k_{0}=k_{0}(\omega)=\sqrt[4]{\frac{2 \rho h \omega^{2}-\beta}{D}}=\sqrt[4]{\frac{2 \rho h}{D}\left(\omega^{2}-\omega_{0}^{2}\right)},
$$

implying that $\omega \geq \omega_{0}$, with

$$
\omega_{0}=\sqrt{\frac{\beta}{2 \rho h}}
$$

denoting the cut-off frequency. The additional condition

$$
k>k_{0}
$$

follows from the assumption of real positive $\lambda_{1}$, which will be clarified further from discussion of the dispersion relation. Substitution of Eq. (7) into the boundary conditions (3) and (4) leads to

$$
\lambda_{1}^{2} \lambda_{2}^{2}+2(1-\nu) \lambda_{1} \lambda_{2}-\nu^{2}=0
$$

Using the expressions (8) and (9), Eq. (13) may be transformed to give the dispersion relation

$$
D k^{4} \gamma_{e}^{4}=2 \rho h \omega^{2}-\beta,
$$

where

$$
\gamma_{e}=\left[(1-\nu)\left(3 \nu-1+2 \sqrt{2 \nu^{2}-2 \nu+1}\right)\right]^{1 / 4}
$$

is a well-known constant for the bending edge wave, ${ }^{1,3}$ depending on the Poisson's ratio only, note that $\gamma_{e}<1$ for all $0<\nu \leq 1$. It may be now observed from the dispersion relation (14) that

$$
k=\frac{1}{\gamma_{e}} k_{0} \quad \text { for } \omega \geq \omega_{0},
$$

therefore the values of $\lambda_{1}$ and $\lambda_{2}$ may be written as

$$
\begin{aligned}
& \lambda_{1}=\sqrt{1-\gamma_{e}^{2}}, \\
& \lambda_{2}=\sqrt{1+\gamma_{e}^{2}},
\end{aligned}
$$

clearly implying that both $\lambda_{1}$ and $\lambda_{2}$ are real and positive, thus ensuring decay away from the edge $y=0$.
In absence of a Winkler foundation $(\beta=0)$ the derived dispersion relation transforms to the well-known dispersion relation for edge waves on a free plate, see Refs. 1 and 3 . We also mention that the relation (14) has the same form as the dispersion relation for the bending wave on an elastically supported beam, e.g., see Ref. 7.

The dispersion relation (14) can be rewritten in dimensionless form as

$$
K^{4}=\Omega^{2}-1,
$$

where

$$
\begin{aligned}
& K=k \gamma_{e} \sqrt[4]{\frac{D}{\beta}}, \\
& \Omega=\omega \sqrt{\frac{2 \rho h}{\beta}} .
\end{aligned}
$$

In this case the dimensionless value of the cut-off frequency is $\Omega=\Omega_{0}=1$, see Fig. 2 .

It is remarkable that due to the presence of the Winkler foundation, the phase velocity normalized by $\sqrt{2 \rho h} / \gamma_{e} \sqrt[4]{\beta D}$,

$$
V^{p h}=\frac{\Omega}{K}=\frac{\Omega}{\sqrt[4]{\Omega^{2}-1}}
$$

has a local minimum $V^{p h}=\sqrt{2}$ at $\Omega=\sqrt{2}$, corresponding to $K=1$. Moreover, at this point $V^{p h}$ coincides with the group velocity

$$
V^{g}=\frac{d \Omega}{d K}=\frac{2 \sqrt[4]{\left(\Omega^{2}-1\right)^{3}}}{\Omega},
$$

see Fig. 3.

It is also worth mentioning that the minimum value $V^{p h}=V^{g}=\sqrt{2}$, associated with the dispersion relation (19), corresponds to the critical speed of a moving load in the 1D classical problem for a beam resting on a Winkler foundation. ${ }^{6}$ Therefore, we may expect the same resonant effect of an edge moving load on an elastically supported plate.

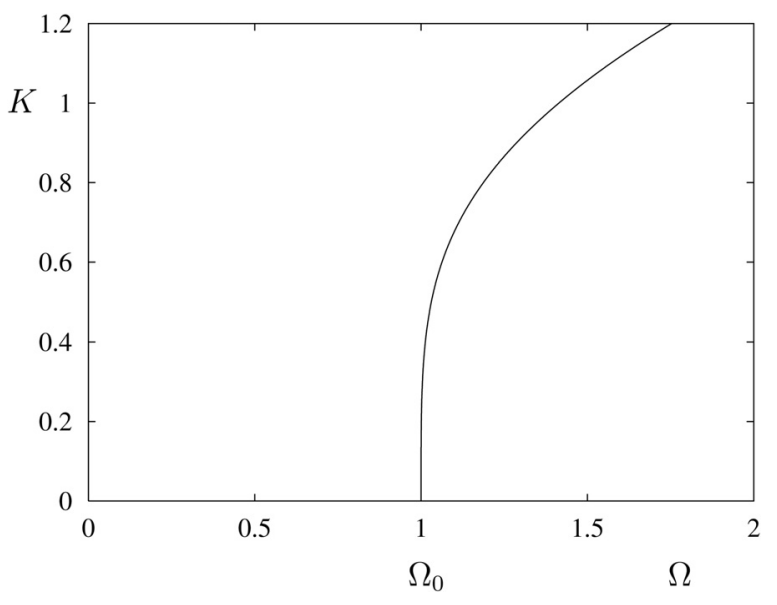

FIG. 2. Dispersion curve for edge wave. 


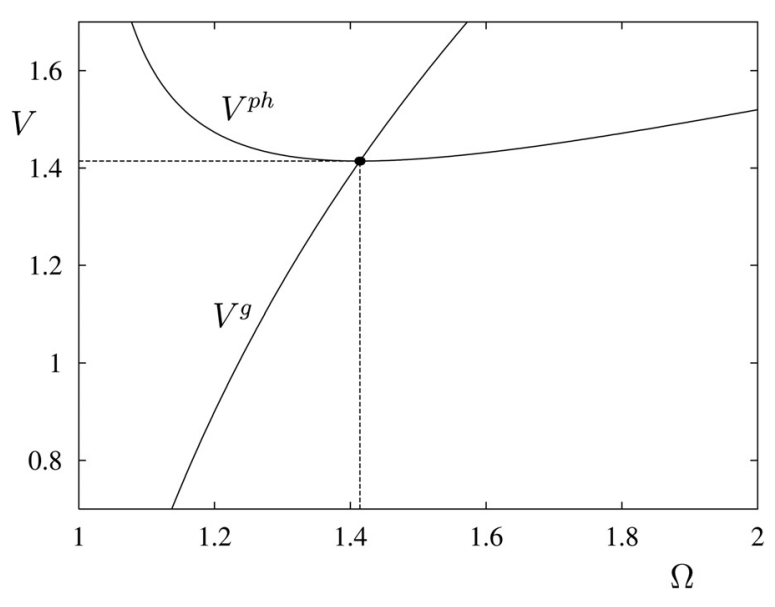

FIG. 3. Phase and group velocities of edge wave.

\section{MOVING LOAD PROBLEM}

Let us replace Eq. (3) by the inhomogeneous boundary condition

$$
\frac{\partial^{2} W}{\partial y^{2}}+\nu \frac{\partial^{2} W}{\partial x^{2}}=M_{0} \delta(x-v t),
$$

corresponding to a concentrated point moment of amplitude $M_{0}$ moving along the edge of the plate at a constant speed $v$.

We now consider the steady-state solution of the problem described by Eqs. (1), (4), and (24) in the moving coordinate system $(\xi=x-v t, y)$. On applying the Fourier transform with respect to $\xi$, we obtain the following boundary value problem for the transformed edge deflection:

$$
\frac{d^{4} W^{F}}{d y^{4}}-2 \alpha^{2} \frac{d^{2} W^{F}}{d y^{2}}+\left(\alpha^{4}-\frac{2 \rho h v^{2}}{D} \alpha^{2}+\frac{\beta}{D}\right) W^{F}=0,
$$

subject to

$$
\begin{aligned}
& \frac{\partial^{2} W^{F}}{\partial y^{2}}-\nu \alpha^{2} W^{F}=M_{0}, \\
& \frac{\partial^{3} W^{F}}{\partial y^{3}}-(2-\nu) \alpha^{2} \frac{\partial W^{F}}{\partial y}=0,
\end{aligned}
$$

where $\alpha$ is the Fourier parameter. The solution of Eqs. (25) and (26) for $W^{F}$ at the edge $y=0$ may be found in the form

$$
\left.W^{F}\right|_{y=0}=\frac{M_{0}\left(\nu \alpha^{2}+q\right)}{q^{2}+2(1-\nu) \alpha^{2} q-\nu^{2} \alpha^{4}},
$$

with

$$
q=\sqrt{\alpha^{4}+\frac{\beta-2 \rho h v^{2} \alpha^{2}}{D}} .
$$

In terms of the dimensionless parameter $A=\alpha \gamma_{e} \sqrt[4]{D / \beta}$, the transformed edge deflection (27) becomes

$$
\left.W^{F}\right|_{y=0}=\frac{P}{\beta\left(A^{4}-V^{2} A^{2}+1\right)},
$$

with

$$
P=P(A, V)=\frac{M_{0} \sqrt{\beta D}\left(\nu A^{2}+Q\right)\left(\chi A^{2}+Q\right)}{\gamma_{e}^{2}\left(Q-A^{2}(2-2 \nu+\chi)\right)},
$$

where

$$
\begin{aligned}
& Q=Q(A, V)=\sqrt{A^{4}-\gamma_{e}^{4}\left(V^{2} A^{2}-1\right)}, \\
& \chi=\sqrt{2 \nu^{2}-2 \nu+1}+\nu-1 .
\end{aligned}
$$

In the formulas above, $V=v \sqrt{2 \rho h} / \gamma_{e} \sqrt[4]{\beta D}$ is the dimensionless speed of the load.

It may be observed that when $V=\sqrt{2}$, the denominator in Eq. (29) has the double poles at $A= \pm 1$. Thus, the critical speed of the load coincides with the minimal value of the phase velocity determined in Sec. II. It is also clear that the near-resonant response for $|V-\sqrt{2}| \ll 1$ is dominated by the contribution arising from the close poles of the aforementioned denominator. In this case, we may approximate the Fourier transform (29) by a simpler expression, substituting the function $P$ by its value $P^{*}=P(1, \sqrt{2})$. This transform corresponds to a 1D moving load problem for a beam resting on a Winkler foundation, see Fig. 4. It is given by the equation, see, e.g., Ref. 4,

$$
D^{*} \frac{\partial^{4} W}{\partial x^{4}}+2 \rho h \frac{\partial^{2} W}{\partial t^{2}}+\beta W=P^{*} \delta(x-v t),
$$

where

$$
\begin{aligned}
& D^{*}=D \gamma_{e}^{4}, \\
& P^{*}=\frac{M_{0} \sqrt{\beta D} \chi(\chi+\nu)}{\gamma_{e}^{2}(\chi+1-\nu)}
\end{aligned}
$$

denote the effective stiffness and amplitude of the point load, respectively. It can be readily verified that the transformed solution of Eq. (33) is given by Eq. (29) provided that $P=P^{*}$.

\section{CONCLUDING REMARKS}

The presence of a Winkler foundation has a significant influence on edge bending waves, including non-monotonic behavior of the phase velocity which causes a resonant regime for a load moving along the plate's edge.

The similarity of the studied wave with a wave on an elastically supported beam concurs with the parabolic-

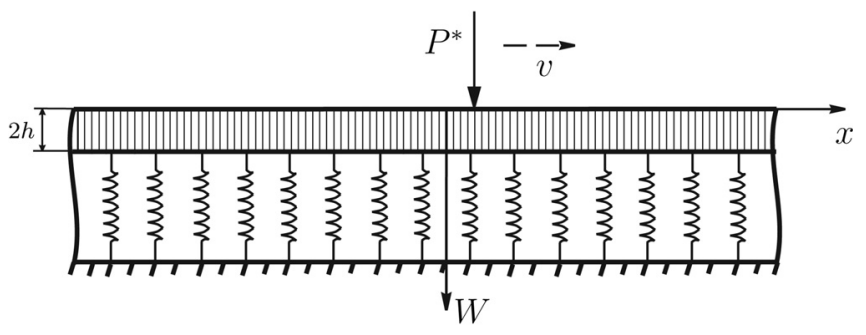

FIG. 4. Moving load problem for a beam on elastic foundation. 
elliptic theory for edge bending waves recently reported in Ref. 8. It is also in line with the earlier established analogy between Rayleigh surface waves and waves on a string. 9,10

The framework of the paper can be extended to anisotropic plates ${ }^{11}$ and also adapted for plates with curved edges. ${ }^{12}$ In addition, there is clear potential for refining the Kirchhoff theory described in Refs. 2 and 13 using the socalled theory with modified inertia (see Ref. 14, and references therein).

${ }^{1}$ Yu. K. Konenkov, "A Rayleigh-type bending wave," Sov. Phys. Acoust. 6, 122-123 (1960).

${ }^{2}$ A. N. Norris, V. V. Krylov, and I. D. Abrahams, "Flexural edge waves and comments on "A new bending wave solutions for the classical plate equation'," J. Acoust. Soc. Am. 107, 1781-1784 (2000).

${ }^{3}$ J. B. Lawrie and J. Kaplunov, "Edge waves and resonance on elastic structures: An overview," Math. Mech. Solids 17(1), 4-16 (2012).

${ }^{4}$ L. Fryba, Vibration of Solids and Structures Under Moving Loads (Noordhoff International, Groningen, The Netherlands, 1972).

${ }^{5}$ Y. H. Wang, L. G. Tham, and Y. K. Cheung, "Beams and plates on elastic foundations: A review," Prog. Struct. Eng. Mater. 7(4), 174-182 (2005).
${ }^{6} \mathrm{~S}$. P. Timoshenko, "Method of analysis of statical and dynamical stresses in rail," in Proceedings of the Second International Congress of Applied Mechanics, Zurich, September 1926 (Orell Fössli Verlag, Zurich, 1927), pp. 407-418.

${ }^{7}$ K. F. Graff, Wave Motion in Elastic Solids (Dover, New York, 1975).

${ }^{8}$ J. Kaplunov and D. A. Prikazchikov, "Explicit models for surface, interfacial and edge waves," in Dynamic Localization Phenomena in Elasticity, Acoustics and Electromagnetism, Vol. 547 of CISM Lecture Notes (Springer-Verlag, Vienna, 2013), pp. 73-114.

${ }^{9}$ J. Kaplunov, A. Zakharov, and D. A. Prikazchikov, "Explicit models for elastic and piezoelastic surface waves," IMA J. Appl. Math. 71, 768-782 (2006).

${ }^{10}$ J. Kaplunov, E. Nolde, and D. A. Prikazchikov, "A revisit to the moving load problem using an asymptotic model for the Rayleigh wave," Wave Motion 47, 440-451 (2010).

${ }^{11}$ A. N. Norris, "Flexural edge waves," J. Sound Vib. 174, 571-573 (1994).

${ }^{12}$ M. Destrade and Y. B. Fu, "A wave near the edge of a circular disk," Open Acoust. J. 1, 15-18 (2008).

${ }^{13}$ D. D. Zakharov, "Analysis of the acoustical edge bending mode in a plate using refined asymptotics," J. Acoust. Soc. Am. 116(2), 872-878 (2004).

${ }^{14}$ J. D. Kaplunov, E. V. Nolde, and B. F. Shorr, "A perturbation approach for evaluating natural frequencies of moderately thick elliptic plates," J. Sound Vib. 281, 905-919 (2005). 